\title{
Functional significance of coronary collateral circulation during dynamic exercise evaluated by thallium-20I myocardial scintigraphy
}

\author{
R J WAINWRIGHT, $M$ N MAISEY, A C EDWARDS, E SOWTON \\ From the Department of Cardiology and the Department of Nuclear Medicine, Guy's Hospital, London
}

SUMMARY Sixty-five patients with angiographically documented coronary artery disease were investigated by thallium-201 ( $\left.{ }^{201} \mathrm{Tl}\right)$ scintigraphy to determine the role of the collateral circulation during dynamic exercise. Fifty-three patients had complete proximal occlusion of at least one major coronary artery. One patient had total occlusion of all three major coronary arteries. Sixty-four collateral channels were identified, graded, and compared with corresponding regions of the myocardial scintigram. Tracer uptake was also graded and classified as various degrees of protection from ischaemia.

A significant correlation between good collaterals with complete protection and poor or absent collaterals with no protection was noted.

Seventeen patients ( 20 occluded vessels) had total coronary occlusion without myocardial infarction. Collaterals conferred protection in $9 / 15$ occlusions whereas no protection was seen in five occlusions without collaterals.

There was no difference in the protective role of homocoronary and heterocoronary collateral vessels. Hypertrophy of the first septal left anterior descending perforator conferred significant protection from ischaemia in contrast to bridging collaterals and ghosting.

During exercise the right coronary bed is preferentially protected from ischaemia, in contrast to the left anterior descending territory. This probably reflects the direction of a transmural flow gradient between left and right ventricles during exercise.

Controversy concerning the functional significance of the coronary collateral circulation in man has been compounded rather than resolved since the introduction of selective coronary arteriography despite the excellent in vivo description of collateral vessels given by several authors using this technique. ${ }^{1-3}$ Perhaps debate is inevitable when no reference method exists for determining collateral flow in man and observations are drawn from indirect measurement. However, two factors deserve emphasis when the protective role of intercoronary anastomoses is considered.

(1) Selection of patients and experimental design. Patients with severe coronary artery disease may be excluded from analysis in two important ways; they may remain undetected and symptom free as a result of well-functioning coronary collaterals, or they may die because of inadequate development of coronary collateral vessels.

Received for publication 22 February 1979
In addition to this inevitable preselection, false comparisons are frequently made between other patients. Thus, patients with coronary collaterals have worse disease than those without, but comparison between these groups should properly be based on equally severe coronary disease. Unfortunately, 'equally severe coronary disease' determined by arteriography does not necessarily indicate equivalent degrees of myocardial ischaemia. The investigator may achieve more homogeneity in selecting severe disease by studying patients with at least complete occlusion of one major coronary artery rather than accept patients with patent and less severely diseased coronary vessels as did Helfant. ${ }^{4}$

(2) Inappropriate methodology. Although coronary arteriography provides clear definition of significant disease in proximal coronary arteries it is less satisfactory in the delineation of coronary collateral vessels for it reveals only epicardial anastomoses of a size no smaller than 100 to $200 \mu$ 
and not the deeper connections which exist within the myocardium. ${ }^{5}$ Moreover, the demonstration that coronary collaterals can be filled with contrast medium does not guarantee their function, despite the claim by Frick et al. ${ }^{6}$ that only collaterals carrying flow are visualised during selective arteriography, always provided that contrast medium is not forced under pressure into the coronary tree.

Myocardial imaging using thallium-201 ( ${ }^{201} \mathrm{Tl}$ ) is a non-invasive technique which is valuable in the analysis of regional myocardial perfusion.? Regions of ischaemia caused by severe coronary disease appear as functional defects of tracer accumulation in the myocardial scintigram. ${ }^{8}$ Using ${ }^{201} \mathrm{Tl}$ imaging, we have analysed the role of the coronary collateral circulation in the preservation of regional myocardial perfusion during angina pectoris induced by dynamic exercise and have identified the angiographic features of collateral vessels responsible for such protection.

\section{Patients and methods}

Sixty-five patients (62 men and three women; mean age 50 years; range 27 to 65 years) with angina pectoris were investigated by ${ }^{201} \mathrm{Tl}$ myocardial scintigraphy shortly after coronary artery disease had been demonstrated by contrast arteriography.

All patients selected for myocardial imaging had severe stenosis or total occlusion of at least one major coronary artery. The majority of patients had complete occlusion of at least one vessel and were homogeneous in this respect. A significant coronary stenosis was defined as reduction of the coronary lumen by more than 50 per cent, and severe stenosis as a reduction in diameter greater than 80 per cent. Selective coronary arteriography was performed in multiple projections using either the Sones or Judkins technique. All patients had a complete physical examination, resting 12 lead electrocardiogram, and an electrocardiogram recorded during a maximal exercise test on a Monark bicycle ergometer with lead V5 monitoring.

Subjects with hypertension, valvular heart disease, or primary myocardial disease were excluded from this study. Drugs for heart disease, particularly beta-blockers, were withdrawn before contrast angiography and myocardial scintigraphy.

During the exercise test each patient received $1.5 \mathrm{mCi}$ of ${ }^{201} \mathrm{Tl}$ through an indwelling intravenous cannula at the onset of angina pectoris or limiting dyspnoea. The exercise end-point was maintained for one and a half to two minutes so that there was maximal myocardial uptake of circulating tracer in the presence of symptoms.

After a 10-minute recovery period, myocardial imaging was performed with subjects in the supine position using an Ohio-Nuclear Series 100 scintillation camera and a high-sensitivity parallel-hole collimator (Fig. 1). Anterior, left anterior oblique $45^{\circ}$ and $55^{\circ}$, and full left lateral projections were routinely acquired in every patient. In each image 200000 counts were collected using a 20 per cent window centred on the $72.5 \mathrm{keV}$ mercury $x$-ray peak emitted from ${ }^{201} \mathrm{Tl}$ decay. During acquisition of the scintigrams activity from background organs such as the liver and lungs was reduced by surrounding the heart with an annulus of lead cape positioned over the praecordium beneath the gamma camera. Each image was acquired on transparency film in
Fig. 1 Myocardial imaging of patient at rest shortly after ${ }^{201} \mathrm{Tl}$ was administered during maximal exercise. Scintillation camera is closely apposed to praecordium while anterior scintigram is acquired. Computer terminal seen in far background links camera to dedicated minicomputer not shown.




four to eight minutes and simultaneously stored in a $64 \times 64$ matrix on a magnetic disc using a dedicated minicomputer (DEC Gamma II) interfaced to the gamma camera. The digital images were enhanced by a 50 per cent background erase, interpolated and displayed on a TV monitor in $\mathbf{1 6}$ grades of colour which were linearly related to image intensity.

Analysis was performed by dividing the scintigram into seven roughly equal segments around its circumference using a light marker controlled by a joy-stick at the computer console. These segments were expressed as average counts per digital matrix element and compared with a normal myocardial profile previously established in our laboratory from ${ }^{201} \mathrm{Tl}$ scintigrams obtained in a group of patients without evidence of heart disease. Segments falling below the established normal range were regarded as myocardial defects.

Each defect was further classified as moderate or severe according to the degree of tracer deficit seen in that region. Moderate defects were defined as a regional reduction in count rate of 50 to 80 per cent and were identified in the colour image as areas containing a predominance of orange or red. This appearance was considered to represent partial protection from ischaemia if it occurred in an area subtended by severe coronary artery disease. A severe uptake defect was defined as a reduction in regional count rate greater than 80 per cent and appeared as a blue or black region in the colour display of the image. These defects were regarded as showing no protection from ischaemia if they were caused by severe coronary artery disease. Normal tracer uptake in regions of the scintigram supplied by severely diseased coronary arteries was regarded as complete protection from ischaemia and appeared as regions of yellow in the colour display image. Fig. 2 shows examples of these appearances.

All scintigrams were reviewed independently by two observers without knowledge of the coronary anatomy and compared with the quantitative segmental analysis. Excellent agreement was found between the two methods ( $>90 \%$ ) and interobserver agreement was similarly high. Observer disagreements were resolved by subsequent review and consensus, calling on a third observer if necessary.

All coronary arteriograms were reviewed independently by two observers in ignorance of the scintigraphic analysis, and note was made of the site and number of complete coronary occlusions and severe coronary stenoses together with any associated coronary collateral channels.

Coronary collateral vessels were divided angiographically into two main types: (a) predominantly single channels opacified by contrast medium injected into a homolateral or contralateral coronary artery, filling via visible or invisible feeding conduits and joining the distal portion of arteries affected by severe proximal coronary disease; (b) visible or invisible 'bridging' collaterals which connect the dislocated ends of a major coronary artery through which the distal segment of the vessel was opacified after injection of contrast medium into the proximal segment.

In addition 'ghosting' of coronary vessels was noted, that is severely delayed anterograde filling and faint opacification of a distal coronary vessel after injection of contrast medium into its proximal portion.

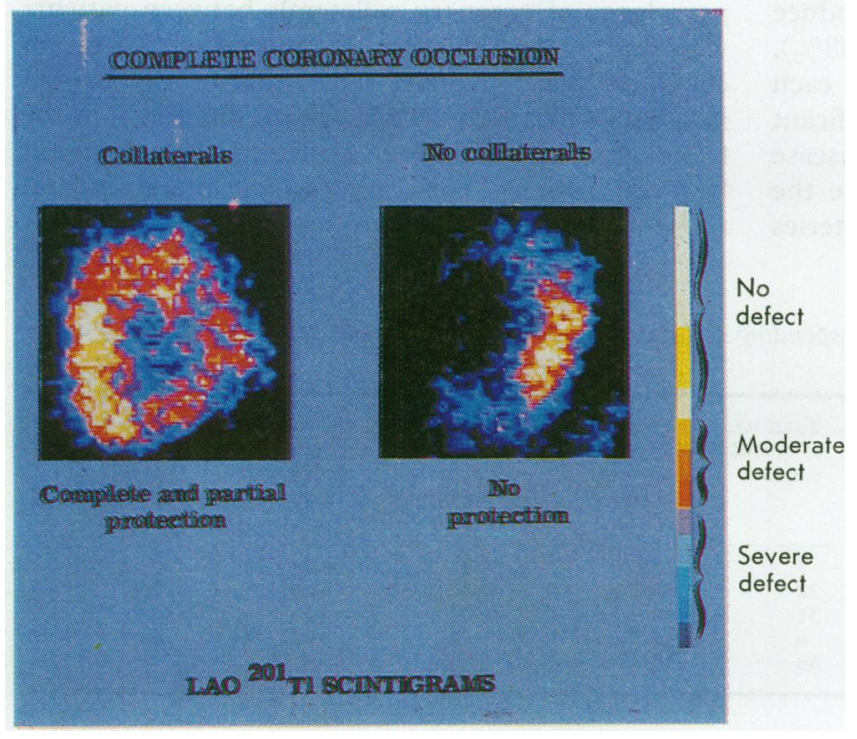

Fig. 2 Left anterior oblique $45^{\circ}$ exercise ${ }^{201} \mathrm{Tl}$ scintigrams. Normal uptake of tracer in septum (IVS) with moderate but extensive defect in inferior wall (IW) and posterolateral wall (PLW) seen in patient with complete occlusion of all three major coronary arteries (left). IVS is yellow indicating complete protection from ischaemia whereas IW and PLW are orange-red signifying partial protection from ischaemia.

Severe defects are seen in IVS and IW in scintigram (right). IVS is black and IW blue indicating no protection from ischaemia.

Sixteen grade colour table displayed (far right) with top of scale corresponding to regions of maximal count rate. 
Heterocoronary and homocoronary collateral vessels were graded angiographically on a five-point ordinal scale according to their promptness and intensity of opacification with contrast medium.

Grade 0: absence of collateral vessels

Grade 1: poorly developed collateral vessels with no prominent distal channel visualised

Grade 2: moderate collaterals giving faint but delayed opacification of a prominent distal channel

Grade 3: good collaterals giving clear opacification of a prominent distal channel

Grade 4: excellent collaterals giving full and brisk opacification of prominent distal vessels.

Agreement between observers was excellent. Of allotted grades, 75 per cent were identical and 92 per cent differed by no more than one category in the scale. No assessment differed by more than two grades and the small number of classifications which differed by more than one grade was resolved by combined review and consensus.

Statistical comparison of data was performed using the $x^{2}$ test corrected for continuity and the Fisher exact test.

\section{Results}

In the 65 patients studied, 110 coronary lesions were classified as angiographically severe and therefore liable to provoke the growth of collateral vessels. There were no significant lesions in the left main coronary artery. Though the majority of patients $(85 \%)$ had developed at least one collateral network at the time of investigation, the observed number of collateral channels in relation to the number of coronary lesions expected to produce collateral development was only $64 / 110$ (58\%). The proportion of collateral channels for each severe coronary lesion showed a highly significant reduction $(P<0.005)$ as single vessel disease progressed to quadruple vessel disease despite the proportion of totally occluded coronary arteries remaining constant in each group (Table 1). This observation suggests that an important factor for the development of adequate collateral growth in response to severe coronary disease and myocardial hypoxia may be an uncompromised blood supply from a healthy donor vessel.

Complete occlusion of at least one major coronary artery was seen in 53 patients: 68 occluded vessels were found in these patients and the prevalence of collateral channels was 78 per cent (Table 2). The right coronary artery was the vessel occluded most commonly ( $50 \%$ of all occlusions) and was also the most frequent recipient of a collateral vessel.

One patient had total proximal occlusion of all three major coronary arteries with no historical or electrocardiographic evidence of myocardial infarction and preservation of excellent left ventricular function. Indeed, a normal resting electrocardiogram, despite total proximal occlusion of at least one large coronary artery, was found relatively often, occurring in $18 / 65(28 \%)$ patients in this study. Twelve patients had at least one severe coronary lesion without complete occlusion of a vessel. The prevalence of coronary collateral channels in this group was similar to that in patients with complete coronary occlusion.

\section{MYOCARDIAL INFARCTION}

Fig. 3 summarises the relation between myocardial infarction and the presence of coronary collaterals. Thirty-nine myocardial infarctions (37 transmural infarcts and two anterior subendocardial infarcts) were documented in 37 patients, an overall prevalence of $39 / 65(60 \%)$.

There was no significant difference in the prevalance of coronary collaterals between patients who had sustained anterior myocardial infarction compared with patients with inferior myocardial infarction. Similarly, there was no difference in the frequency of coronary collateral development between patients with myocardial infarction and those without infarction.

Table 1 Distribution of occluded arteries and corresponding collateral vessels in patients with severe coronary artery disease

\begin{tabular}{|c|c|c|c|c|c|c|c|c|}
\hline $\begin{array}{l}\text { No. of vessels } \\
\text { with significant } \\
\text { disease (stenosis } \\
>50 \% \text { ) }\end{array}$ & $\begin{array}{l}\text { No. of } \\
\text { patients }\end{array}$ & $\begin{array}{l}\text { No. of patients } \\
\text { with at least } \\
\text { one collateral } \\
\text { bed }\end{array}$ & $\begin{array}{l}\text { Total no. } \\
\text { of collateral } \\
\text { beds }\end{array}$ & $\begin{array}{l}\text { Total no. } \\
\text { of occluded } \\
\text { vessels }\end{array}$ & $\begin{array}{l}\text { Total no. of } \\
\text { severe lesions } \\
\text { (stenosis } \\
>80 \% \text { ) }\end{array}$ & $\begin{array}{l}\text { Proportion of } \\
\text { collateral } \\
\text { beds per } \\
\text { severe lesion }\end{array}$ & $\begin{array}{l}\text { Proportion of } \\
\text { occluded vessels } \\
\text { per severe } \\
\text { lesion }\end{array}$ & $\begin{array}{l}\text { Proportion of } \\
\text { collateral beds } \\
\text { associated } \\
\text { with occluded } \\
\text { vessels }\end{array}$ \\
\hline $\begin{array}{l}\text { Single } \\
\text { Double } \\
\text { Triple } \\
\text { Quadruple } \\
\text { Total }\end{array}$ & $\begin{array}{r}13 \\
21 \\
26 \\
5 \\
65\end{array}$ & $\begin{array}{r}9 \\
21 \\
21 \\
4 \\
55\end{array}$ & $\begin{array}{r}9 \\
27 \\
34 \\
4 \\
64\end{array}$ & $\begin{array}{r}7 \\
22 \\
31 \\
8 \\
68\end{array}$ & $\begin{array}{r}13 \\
32 \\
52 \\
13 \\
110\end{array}$ & $\begin{array}{l}0.69 \\
0.84 \\
0.65 \\
0.31 \text { ॠ }\end{array}$ & $\begin{array}{l}0.54 \\
0.69 \\
0.60 \\
0.62\end{array}$ & $\begin{array}{l}0.62 \\
0.90 \\
0.62 \\
0.60\end{array}$ \\
\hline
\end{tabular}

$\star P<0.0005$. 
Individual coronary arteries and their collateral supply

Left anterior descending collaterals. No patient with left anterior descending collaterals of any category had complete protection from the appearance of exercise-induced uptake defects in the ${ }^{201} \mathrm{Tl}$ scintigram, though seven patients did exhibit varying degrees of partial protection. Only one patient in the partially protected group had poor development of collateral vessels compared with 19 patients in the group with no protection $(P<0.01)$ (Fig. 4). Hypertrophy of the first septal perforating artery was included in the analysis of left anterior descending collaterals and classified angiographically as a grade 3 (good) collateral vessel in view of its large calibre lumen and rapid filling. Enlargement of this vessel appeared to provide significant protection from ischaemia in the septal distribution of the left anterior descending. Four of seven patients in the group with partial left anterior descending uptake defects had hypertrophy of the first septal perforator compared with only one of 27 patients in the group with no protection as seen on the scintigram $(P=0.003)$.

Right coronary artery collaterals. All three grades of scintigraphic protection were seen in patients with right coronary artery collaterals (Fig. 5). Twelve patients with total proximal occlusion (10 patients) or severe stenosis (two patients) of a dominant right coronary artery had complete protection from ischaemia as shown by completely normal myocardial uptake of ${ }^{201} \mathrm{Tl}$ in the distribution of this vessel. It was surprising to see this degree of

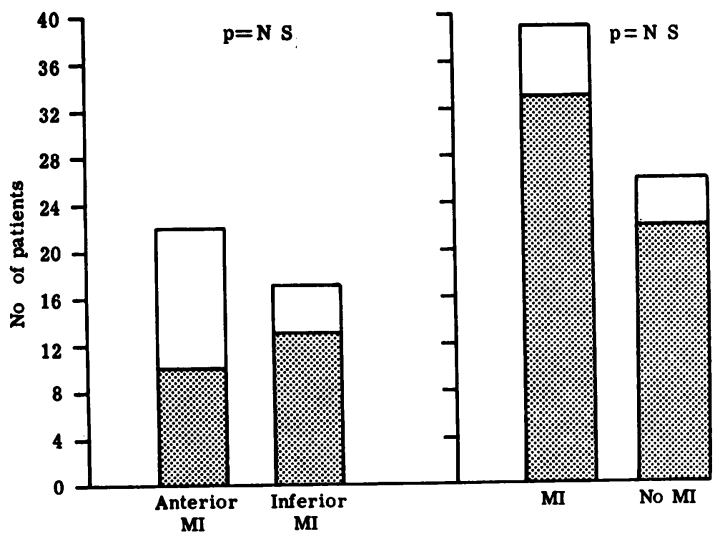

Collaterals

Fig. 3 Prevalence of coronary collaterals was not significantly different in patients with anterior and inferior myocardial infarction (left), nor was there significant difference in prevalence of collaterals between patients with myocardial infarction and patients without myocardial infarction (right).

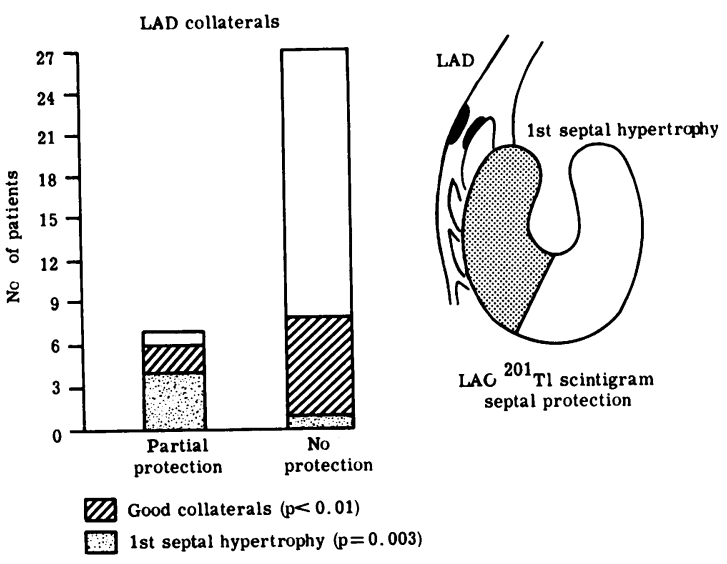

Fig. 4 Patients with severe left anterior descending disease and partial protection in corresponding regions of scintigram have higher prevalence of adequate collateral channels than patients with no scintigraphic protection. Most patients have no protection despite presence of collaterals. No patient has complete protection.

Hypertrophy of first septal perforator is significant factor in preservation of septal tracer uptake.

protection in the presence of extensive disease in other coronary vessels, for example nine patients had severe triple vessel disease while the artery donating collaterals to the right coronary artery had significant disease in 11 patients.

The prevalence of collaterals allotted grade 3 or more was highest in patients with complete protection in the scintigram and fell with decreasing degrees of protection, though these figures did not achieve statistical significance $(P>0.5)$. No patient with complete protection had absent collateral development in contrast to four patients with partial or no protection who had no collateral channels visible on angiography.

In six patients with no scintigraphic protection, four had sustained inferior myocardial infarction despite visualisation of good collaterals to the right coronary artery in three. Eight inferior myocardial infarctions were found in the partially protected group while no patient with myocardial infarction was seen in the group with complete protection $(P<0 \cdot 1)$.

Left circumflex collaterals. Left circumflex coronary lesions were not often associated with prominent retrograde collateral filling though there was often angiographic 'ghosting' of the distal bed. Fig. 6 shows that the majority of patients with severe left circumflex coronary lesions had no protection from the appearance of uptake defects in the myocardial scintigram seen in the distribution of this vessel. No patient with this lack of protection had adequate development of collateral channels, whereas three 
patients with partial protection had moderately good collateral growth. One patient with excellent retrograde visualisation of the whole left circumflex artery had entirely normal tracer uptake in the distribution of this vessel. Thus, poor collaterals and ghosting of the left circumflex territory were significantly associated with prominent uptake defects in the ${ }^{201} \mathrm{Tl}$ myocardial scintigram and did not confer protection $(P=0.05)$.

The influence of coronary collateral channels on the myocardial uptake of ${ }^{201} \mathrm{Tl}$ may be demonstrated more clearly by examining only regions of ischaemia and excluding regions of necrosis, for myocardial infarction causes permanent defects of ${ }^{211} \mathrm{Tl}$ accumulation which cannot be attenuated by subsequent revascularisation.

\section{PATIENTS WITH CORONARY OCCLUSION WITHOUT MYOCARDIAL INFARCTION}

Only 17 patients had no historical or electrocardiographic evidence of myocardial infarction and total occlusion of at least one major coronary artery. The scintigrams of these patients were correlated with the appearance of corresponding coronary collateral vessels seen on angiography. Twenty occluded coronary vessels were found in this group of patients, most of whom had one artery completely occluded and significant disease in other vessels. Six patients had left anterior descending artery occlusion, seven patients had right coronary artery occlusion, and two patients had left circumflex artery occlusion. One patient had two vessels completely occluded and another patient had all three coronary arteries occluded without myocardial infarction.

Fig. 7 shows that the presence of collateral vessels confers significant protection against the appearance of exercise-induced ischaemic defects in the ${ }^{201} \mathrm{Tl}$ scintigram. In the presence of collaterals nine of 15 ischaemic vascular beds were associated with mild or no uptake defects whereas all five vascular beds (two left anterior descending and three left circumflex) without a collateral circulation were readily identified as stark defects appearing in corresponding regions of the scintigram $(P=0.03)$. There was no significant difference in the prevalence of severe coronary disease between these groups nor was there

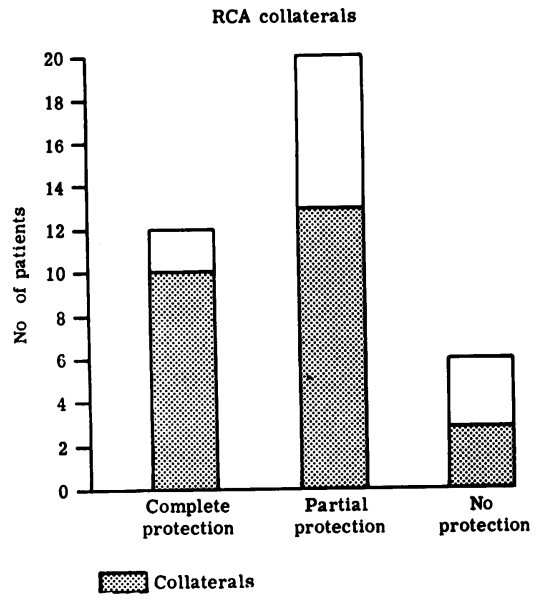

Fig. 5 Twelve patients have normal uptake of ${ }^{201} \mathrm{Tl}$ (complete protection) in distribution of right coronary artery despite severe occlusive disease in this vessel. Ten of these patients have excellent development of coronary collateral vessels though there is no significant difference in prevalance of collaterals among degrees of protection.

any significant difference in the total exercise achieved by the time ${ }^{201} \mathrm{Tl}$ was administered $(3385 \mathrm{kpm} \mathrm{v}$ $3130 \mathrm{kpm}$, NS).

\section{PATIENTS WITH OCCLUSION OF TWO}

CORONARY ARTERIES

Patients with double coronary artery occlusion were of interest in the functional interpretation of dual collateral beds. Retrograde opacification of the distal segment of each occluded vessel after injection of contrast medium into the proximal segment of a diseased donor artery was commonly seen under the artificial pressure gradients produced in the coronary tree at the time of angiography. No information is provided by arteriography about the direction of collateral flow during angina pectoris induced by exercise.

Combined occlusion of the right coronary artery and left anterior descending artery was found in nine out of 13 patients. Anterior myocardial infarction was no more frequent than inferior myocardial infarction in these patients nor was there a difference in the prevalence of coronary

Table 2 Prevalence of collateral vessels in patients with total occlusion of at least one coronary artery

\begin{tabular}{|c|c|c|c|c|c|c|}
\hline \multirow{2}{*}{$\begin{array}{l}\text { No. of occluded vessels } \\
\text { per patient }\end{array}$} & \multirow{2}{*}{$\begin{array}{l}\text { No. of } \\
\text { patients }\end{array}$} & \multirow{2}{*}{$\begin{array}{l}\text { Total no. of collateral } \\
\text { beds }\end{array}$} & \multirow{2}{*}{$\begin{array}{l}\text { Total no. of occluded } \\
\text { vessels }\end{array}$} & \multicolumn{3}{|c|}{ No. of collateral beds per occluded vessel } \\
\hline & & & & $R C A$ & $L A D$ & $L C x$ \\
\hline $\begin{array}{l}1 \\
2 \\
3 \\
\text { Total }\end{array}$ & $\begin{array}{r}39 \\
13 \\
1 \\
53\end{array}$ & $\begin{array}{r}29 \\
22 \\
2 \\
53\end{array}$ & $\begin{array}{r}39 \\
26 \\
3 \\
68\end{array}$ & $\begin{array}{r}17 \\
11 \\
1 \\
29\end{array}$ & $\begin{array}{r}9 \\
8 \\
1 \\
18\end{array}$ & $\begin{array}{l}3 \\
3 \\
0 \\
6\end{array}$ \\
\hline
\end{tabular}

RCA, right coronary artery; LAD, left anterior descending artery; LCx, left circumflex artery. 
collateral channels feeding the distal bed of either the right coronary artery or left anterior descending artery. However, there was a distinct difference in the pattern of scintigraphic uptake defects produced by these two occluded vessels.

Right coronary artery occlusion was associated with significantly less severe uptake defects in the myocardial scintigram than was total occlusion of the left anterior descending $(P<0.05)$ (Table 3$)$. Thus, during maximal dynamic exercise the collateral circulation to the right coronary bed maintained a delivery and distribution of ${ }^{201} \mathrm{Tl}$ sufficient to prevent uptake defects appearing in this region of the scintigram. In contrast, little protective effect could be shown for collaterals supplying the distal left anterior descending, for uptake defects regularly appeared in the distribution of this artery at the limiting end-point of exercise. Defects attributable to right coronary artery disease were never seen in the absence of defects resulting from left anterior descending disease, that is the left anterior descending artery was probably always responsible for producing angina with this combination of coronary occlusions.

A similar situation was seen in patients with

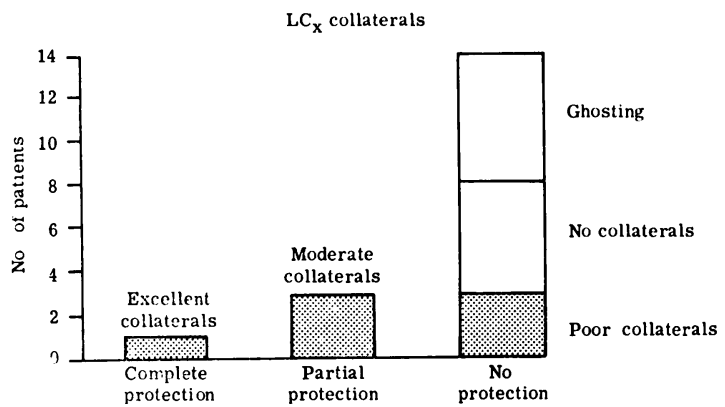

Fig. 6 Most patients with severe disease in left circumflex coronary artery have no protection from ischaemia; in particular, angiographic ghosting and absent collaterals are always accompanied by severe uptake defects in scintigram (no protection). Three patients with moderate collateral development have partial protection from ischaemia and one patient with excellent collateral vessels has complete protection.

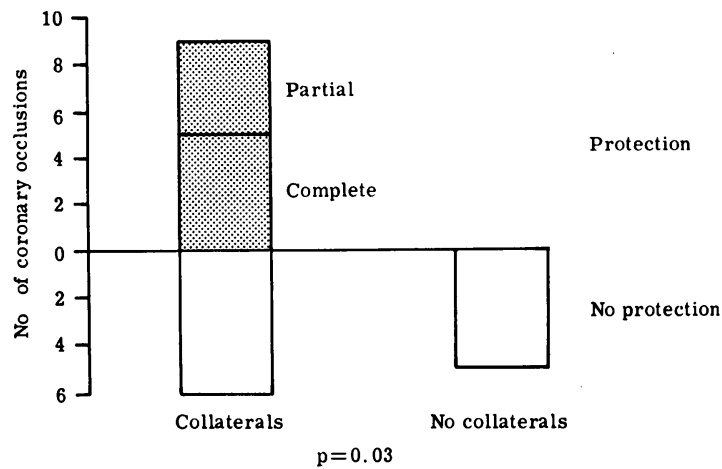

Fig. 7 Collateral development and accompanying protection from ischaemia seen in patients with complete occlusion of at least one vessel but no evidence of myocardial infarction. Protection was never seen in absence of coronary collateral channels.

combined right coronary artery and left circumflex coronary artery occlusion. Myocardial uptake defects were always seen in the distribution of the left circumflex vessel whereas partial defects or no uptake defects at all occurred in the distribution of the right coronary artery.

Only one patient had combined left anterior descending and left circumflex coronary occlusions both of which were apparent in the scintigram, there being a poor collateral supply to each vessel.

CORRELATION OF COLLATERALS WITH ${ }^{201} \mathrm{TL}$ MYOCARDIAL UPTAKE

Fig. 8 shows the relation between the degree of coronary collateral development assessed angiographically and the severity of ${ }^{201} \mathrm{Tl}$ uptake defects seen in the myocardial scintigram. There was a highly significant association between welldeveloped coronary collateral vessels and accompanying preservation of myocardial ${ }^{201} \mathrm{Tl}$ uptake. Similarly, poor or absent coronary collateral development was usually associated with severe deficit of tracer in corresponding regions of the scintigram $(P<0.0005$, coefficient of contingency $=$ $0 \cdot 50$ ).

There was no significant difference in the

Table 3 Prevalence of collateral vessels in patients with complete occlusion of two coronary arteries

\begin{tabular}{|c|c|c|c|c|c|c|c|}
\hline \multirow{2}{*}{$\begin{array}{l}\text { Combinations of coronary artery } \\
\text { occlusion }\end{array}$} & \multirow[t]{2}{*}{ No. of patients } & \multicolumn{3}{|c|}{ No. of collateral beds } & \multicolumn{3}{|c|}{$\begin{array}{l}\text { No. of corresponding protected areas in } \\
\text { scintigram }\end{array}$} \\
\hline & & $R C A$ & $L A D$ & $L C x$ & $R C A$ & $L A D$ & $L C x$ \\
\hline $\begin{array}{l}\text { RCA and LAD } \\
\text { RCA and } L C x \\
L A D \text { and } L C x\end{array}$ & $\begin{array}{l}9 \\
3 \\
1\end{array}$ & $\begin{array}{l}8 \\
3 \\
-\end{array}$ & $\frac{8}{1}$ & $\begin{array}{l}- \\
0 \\
0\end{array}$ & $\begin{array}{l}7^{\star} \\
2 \\
\end{array}$ & $\frac{4}{0}$ & $\begin{array}{l}- \\
0 \\
0\end{array}$ \\
\hline
\end{tabular}

$\star \mathrm{P}<0.005$ for difference in severity between uptake defects found in right coronary artery and left anterior descending artery territories. RCA, right coronary artery; LAD, left anterior descending artery; LCx, left circumflex artery. 
protective effect of homocoronary and heterocoronary collateral vessels independent of their degree of development. Angiographically invisible coronary collaterals were never associated with complete scintigraphic protection and usually conferred no protective benefit. Similarly, vessel ghosting was always accompanied by severe scintigraphic uptake defects. Bridging collaterals also offered inadequate protection from myocardial ischaemia during exercise, but hypertrophy of the first septal perforating artery did appear to offer significant protection to the septum in the presence of severe left anterior descending disease.

\section{Discussion}

The advent of coronary artery bypass graft surgery has brought renewed interest in the coronary collateral circulation and its functional significance, for the chances of successful graft patency are enhanced if the distal segment of an occluded artery has good flow maintained by collateral vessels. Some patients may have sufficient collateral flow to render this operation superfluous though the early study of Blumgart ${ }^{9}$ suggests that collaterals are adequate only at rest. This finding is supported by Helfant and colleagues ${ }^{4}$ who noted that abnormal myocardial lactate metabolism and abnormal postexercise electrocardiograms were even more common in patients with collateral vessels than in those without, which led the authors to conclude that coronary collateral vessels were inadequate to meet the increased myocardial oxygen demands during stress. A similar inadequacy of the collateral circulation to prevent ischaemia, as measured by a submaximal treadmill test, was found by Harris et al. ${ }^{10}$

Using ${ }^{201} \mathrm{Tl}$ myocardial scintigraphy many patients with total occlusive disease of a dominant right coronary artery are seen to have adequate collateral vessels providing complete protection from ischaemia in the distribution of this artery during dynamic exercise. Patients with left anterior descending disease, however, were not protected from the appearance of ischaemic uptake defects. Selective protection of the distal right coronary artery would neither prevent abnormal myocardial lactate metabolism nor abnormal exercise electrocardiograms in the presence of severe left anterior descending disease. Previous studies using these indirect measures of regional myocardial perfusion may have failed to detect the preferential vascular protection now described.

Although the mechanism for this protection is not clear the normal pressure gradient that exists between left and right ventricles may be increased during dynamic exercise, particularly during diastole in an ischaemic left ventricle, so that collateral flow is maintained or increased in the direction of the low pressure right ventricular myocardium. This effect probably accounts for the preservation of tracer uptake in the inferior wall of the left ventricle as seen in ${ }^{201} \mathrm{Tl}$ scintigrams. Clinical support for this concept is found in two situations: (a) right ventricular myocardial infarction is rare in man despite the frequency of total right coronary artery occlusion ${ }^{11}$; (b) internal mammary artery implants to the right ventricle remain patent more easily than implants to the left ventricle. ${ }^{12}$

Experimental support for this concept is also provided by Ramo et al..$^{13}$ who showed that despite similar rates of right coronary artery and left anterior descending coronary occlusion in the pig, collateral vessels to the right coronary artery developed more rapidly and more extensively than to the left anterior descending.

However, one relevant limitation of ${ }^{201} \mathrm{Tl}$ imaging is that relative regional myocardial
Fig. 8 Correlation of coronary collateral development with ${ }^{201} \mathrm{Tl}$ myocardial uptake seen in corresponding regions of the scintigram. I, Homocoronary and heterocoronary collaterals; $B$, bridging collaterals; $\uparrow$, hypertrophy of first septal perforator of left anterior descending artery; + , ghosting.

\begin{tabular}{|c|c|c|c|c|c|}
\hline \multirow[b]{2}{*}{ SCINTIGRAM } & \multicolumn{5}{|c|}{$\begin{array}{l}\text { ANGIOGRAM } \\
\text { Collateral grades }\end{array}$} \\
\hline & Nil & Poor & Moderate & Good & Excellent \\
\hline $\begin{array}{l}\text { Complete } \\
\text { protection }\end{array}$ & & & 1 & 018181 & 1010101 \\
\hline $\begin{array}{l}\text { Partial } \\
\text { protection }\end{array}$ & 101 & 100 & $\left.\right|_{B} ^{10} 1000000$ & $\begin{array}{l}11018 \\
4+4\end{array}$ & 00100 \\
\hline $\begin{array}{l}\text { No } \\
\text { protection }\end{array}$ & 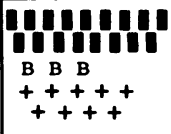 & 10101001 & 10101 & 1 & 1010 1 \\
\hline
\end{tabular}


ischaemia is reflected in the scintigram rather than in terms of absolute perfusion. Thus, it is possible that even the right coronary artery territory is ischaemic during exercise, though better perfused than the left coronary artery.

Several conclusions remain to be drawn from these data:

(a) There is no difference between homocoronary and heterocoronary anastomoses in protection from ischaemia.

(b) Enlargement of the first septal branch of the left anterior descending appears to provide significant protection from septal ischaemia and should be regarded as an important collateral channel.

(c) In contrast to the study of Knoebel et al..$^{14}$ bridging collaterals did not offer any protection from the scintigraphic detection of ischaemia.

(d) Angiographic ghosting of vessels was always associated with severe uptake defects in the scintigram. Ghosting probably occurs because of previous severe occlusive disease and does not indicate a collateral channel of any functional significance.

(e) The clear correlation between the angiographic grading of collaterals and corresponding areas of protection seen in the scintigram gives the angiographer reasonable reassurance that there is functional relevance in a detailed description of these important channels. Further support for this correlation is found in the postmortem data of Spain et al. ${ }^{15}$ and in the study of Scherer et al. ${ }^{16}$ who compared collaterals quantified angiographically before operation with collateral flow measured during coronary artery bypass surgery.

${ }^{201} \mathrm{Tl}$ scintigraphy provides a functional map of the myocardium in terms of patterns of ischaemia and thus allows a functional interpretation of corresponding anatomy seen in the coronary arteriogram. In future it may be possible to assess non-invasively the influence of chronic interventions such as graded physical exercise and drug therapy on the time-course and development of coronary collateral vessels by monitoring changes in the ${ }^{201} \mathrm{Tl}$ scintigram.

Dr R J Wainwright was supported by a British Heart Foundation grant.

\section{References}

'Baroldi G, Mantero O, Scomazzoni G. The collaterals of coronary arteries in normal and pathologic hearts. Circ Res 1956; 4: 223-9.
${ }^{2}$ Gensini GG, Bruto da Costa BC. The coronary collateral circulation in living man. Am $\mathcal{f}$ Cardiol 1969; 24: 393-400.

James TN. The delivery and distribution of the coronary collateral circulation. Chest 1970; 58: 183203.

${ }^{4}$ Helfant RH, Vokonas PS, Gorlin R. Functional importance of the human coronary collateral circulation. N Engl f Med 1971; 284: 1277-81.

${ }^{5}$ Gregg DE. The natural history of coronary collateral development. Circ Res 1974; 35: 335-44.

${ }^{6}$ Frick MH, Valle M, Korhola O, Riihimäki E, Wiljasalo M. Analysis of coronary collaterals in ischaemic heart disease by angiography during pacing induced ischaemia. Br Heart $\mathcal{F} 1976$; 38: 186-96.

'Strauss HW, Harrison K, Langan JK, Lebowitz E, Pitt B. Thallium-201 for myocardial imaging. Relation of thallium-201 to regional myocardial perfusion. Circulation 1975; 51: 641-5.

${ }^{8}$ Bailey IK, Griffith LSC, Strauss HW, Pitt B. Detection of coronary artery disease and myocardial ischemia by electrocardiography and myocardial perfusion scanning with thallium-201 (abstract). Am $\mathcal{F}$ Cardiol 1976; 37: 118.

${ }^{9}$ Blumgart $\mathrm{HL}$, Schlesinger MJ, Davis D. Studies on the relation of the clinical manifestations of angina pectoris, coronary thrombosis, and myocardial infarction to the pathologic findings. Am Heart $\mathcal{F} 1940$; 19: $1-91$.

${ }^{10}$ Harris CN, Kaplan MA, Parker DP, Aronow WS, Ellestad $\mathrm{MH}$. Anatomic and functional correlates of intercoronary collateral vessels. Am $\mathscr{f}$ Cardiol 1972; 30: 611-14.

${ }^{11 W a r t m a n n}$ WB, Hellerstein HK. The incidence of heart disease in 2000 autopsies. Ann Intern Med 1948; 28: 41-65.

${ }^{12}$ Vineberg A, Zannora B. Revascularization of the right ventricular myocardium via right coronary arterial system by right internal mammary artery implantation. Am f Cardiol 1968; 22: 218-26.

${ }^{13}$ Ramo BW, Peter RH, Ratliff N, Kong Y, McIntosh HD, Morris JJ Jr. The natural history of right coronary arterial occlusion in the pig. Comparison with left anterior descending arterial occlusion. Am $\mathcal{F ~ C a r d i o l}$ 1970; 26: 156-61.

${ }^{14}$ Knoebel SB, McHenry PL, Phillips JF, Pauletto FJ. Coronary collateral circulation and myocardial blood flow reserve. Circulation 1972; 46: 84-94.

${ }^{15}$ Spain DM, Bradess VA, Iral P, Cruz A. Intercoronary anastomotic channels and sudden unexpected death from advanced coronary atherosclerosis. Circulation 1963; 27: 12-17.

${ }^{16}$ Scherer JL, Goldstein RE, Stinson EB, Seningen RP, Grehl TM, Epstein SE. Correlation of angiographic and physiologic assessment of coronary collaterals in patients receiving by-pass grafts (abstract). Circulation 1973; 47 and 48: Suppl. IV, IV-88.

Requests for reprints to $\operatorname{Dr} \mathbf{R} J$ Wainwright, Department of Cardiology, Guy's Hospital, London SE1 9RT. 